\title{
Cuando el paciente resulta desagradable, el dolor es subestimado
}

\author{
When the patient is disliked, the pain is underestimated
}

De Ruddere L y col. Pain 2011;152: 2342-47.

\section{Objetivos}

Evaluar la influencia de la simpatía del paciente en la estimación del dolor realizado por profesionales de la salud.

\section{Diseño}

Estudio experimental con control propio y concurrente*.

\section{Lugar}

Universidad de Ghent, Bélgica.

\section{Sujetos}

Cuarenta observadores mayores de 18 años que hablaran el idioma oficial (17 hombres y 23 mujeres) reclutados a través de un aviso de diario. Criterio de exclusión: enfermedad psiquiátrica. La participación fue remunerada. La intención del estudio solo fue revelada al concluir el mismo.

\section{Descripción del experimento}

Fase 1: se expuso a los observadores a un estimulo no condicionado: mirar fotos de seis pacientes provenientes de un archivo de dolor de hombro, y luego a un estímulo condicionado: a cada paciente se les asignaron tres adjetivos de rasgos de personalidad (positivos, neutros o negativos). Fase 2: se mostraron 48 videos a cada observador (ocho fragmentos de cada uno de los seis pacientes) con expresiones faciales producidas durante una evaluación kinesiológica por dolor de hombro y se les pidió que estimaran el grado de dolor. A posteriori se volvió a presentar la foto de los pacientes y se les solicito que puntúen el grado de simpatía por el paciente.

\section{Medición de resultados principales}

Estimación del dolor mediante una escala visual analógica. Estimación del grado de simpatía por el paciente, utilizando tres cuestionarios ad hoc.

\section{Resultados principales}

La estimación del dolor de los pacientes con rasgos negativos fue significativamente más baja que la de los pacientes con rasgos neutros o rasgos positivos.

\section{Conclusiones}

La intensidad del dolor suele infravalorarse en los pacientes que causan desagrado en relación a los pacientes que causan simpatía. Se necesitan estudios adicionales para evaluar los mecanismos asociados a este fenómeno y sus posibles consecuencias.

Fuente de financiamiento: Beca de la Fund for Scientific Research (FWO)Flanders.

\section{Comentario}

El dolor en la consulta ambulatoria es altamente prevalente, tanto como las diferencias de personalidad entre el médico y el paciente.

Revisemos los principales puntos de impacto de este trabajo: a) La exposición a un estímulo condicionado plantea la facilidad con la que nos formamos una imagen de las personas a partir simplemente de asociarlas con ciertas cualidades positivas o negativas. Las referencias que se hagan de la personalidad de los pacientes en el contexto del equipo de salud pueden condicionar al profesional a la hora de trabajar con dicho paciente. b) Considerar una intensidad de dolor diferente a la que sufre el paciente puede constituir un error médico diagnóstico y por ende terapéutico ${ }^{1}$. c) La sensación de conocer el padecimiento del otro constituye el componente cognitivo de la empatía ${ }^{2}$, por lo que una estimación adecuada del dolor también repercute positivamente en el paciente por sentirse comprendido en un grado cercano al de su sufrimiento. d) Ante situaciones que alteren la forma en la que el pro- fesional percibe al paciente, como burn-out o depresión ${ }^{3}$, la posibilidad de menospreciar el dolor podría ser aún mayor. e) Entre las variables que afectan la estimación del dolor están las relacionadas a factores del contexto en el que aparece, como la presencia de una causa "médica". ¿Cuán deficiente puede llegar a ser la apreciación del dolor en pacientes que consultan muy frecuentemente o con síntomas somáticos no explicables, que además se asocian a rasgos negativos de la personalidad? ¿Se puede pensar en extender los resultados de este estudio a otros síntomas?

\section{Conclusiones del comentador}

Cuando la valoración de personalidad de nuestros pacientes es negativa, podemos llegar a aceptarlos menos, incluso a rechazarlos y eso condiciona nuestra habilidad diagnóstica (de intensidad de dolor) y nuestra capacidad empática.

Lila Paula Dupuy [ Médica de familia - Docente Adscripto Universidad Maimonides- OS Luis Pasteurliladupuy @gmail.com ]

Dupuy L. Cuando el paciente resulta desagradable, el dolor es subestimado. Evid Act Pract Ambul Abr-Jun 2012;15(2):53. Comentado de: De Ruddere L, Goubert L, Martin Prkachin K, y col. When you dislike patients, pain is taken less seriously. Pain 2011;152: 2342-47. PMID: 21943961

\section{Referencias}

1. West C, Huschka M, Novotny P, et al. Asociation of perceived medical errors whith residente distress and empathy. JAMA 2006;296(9):1071-8

2. Goubert $L y$ col. Facing others in pain: the effects of empathy. Pain 2005;118:285-8.

3. Krasner $\mathrm{M}$ y col. Association of an educational program in mindful communication with burnout, empathy and attitudes among primary care physicians. JAMA 2009;302(12):1284-93. 